\title{
Spectral Properties of Disordered Systems in the One-Body Approximation
}

\author{
L. A. Pastur \\ Physico-Technical Institute of Low Temperatures, \\ UkrSSR Academy of Sciences, Kharkov, USSR
}

\begin{abstract}
The paper considers the Schrödinger equation for a single particle and its discrete analogues. Assuming that the coefficients of these equations are homogeneous and ergodic random fields, it is proved that the spectra of corresponding random operators and their point spectra are dense with probability 1 and that in the one-dimensional case they have no absolutely continuous component. Rather wide sufficient conditions of exponential growth of the Cauchy solutions of the one-dimensional equations considered are found.
\end{abstract}

\section{Introduction}

Experimental and theoretical investigation of a large variety of disordered systems has constituted in the recent decades a substantial part of the condensed matter physics. One of the most common and efficient theoretical approaches in this field now is the one-body approximation. In this approximation the solution of a problem is reduced to consideration of different quantities constructed of eigenvalues and eigenfunctions of the one-body Schrödinger equation or its discrete analogues (see [1-3] for physical discussion and numerous references, and [4] for the survey and discussion from a more mathematical point of view).

The usual approach which conforms to the general principles of statistical physics is to consider operators defined by these equations and some selfadjoint boundary conditions in a finite domain $V$ and study, for $V \rightarrow \infty$, asymptotic properties of the various physical quantities which are all constructed of eigenelements of corresponding operators.

The random potential that models the influence of a disordered medium should meet the following natural conditions: and

a) spatial homogeneity

b) absence of statistical correlations between infinitely distant points.

In mathematical terms it means that the potential is realization of a random field (a random process in one-dimensional case) which is 
A) homogeneous, and

B) metrically transitive (ergodic),

(for definition see, e.g. [5]).

It turns out that in such case the physical quantities of a macroscopically large disordered system are nonrandom ${ }^{1}$ and can be expressed through the averaged spectral characteristics of the limit operator $H$ acting in the whole $d$-dimensional space $\mathbb{R}^{d}$ (or the lattice $\mathbb{Z}^{d}$ ). This justifies a closer study of this operator which moreover should be expected to reveal the spatial homogeneity more naturally and completely.

The main object of this paper is to study the spectral properties of the operator $H$ acting in the whole space. As we shall $\mathrm{see}^{2}$, many properties are common to the spectra of all such operators, though quite different from those of the ordered counterparts, i.e. difference and differential operators with periodic coefficients.

The paper is organized as follows. In Sect. 2 we introduce and study a certain class of operators (we call them metrically transitive or ergodic) including the difference and differential operators with random ergodic coefficients as an important subclass. Section 3 contains results for the one-dimensional case. In Sect. 4 we study the relationship between the spectral characteristics of the operators acting in the whole space and those of the corresponding operators defined by the same equations in a large but finite domain. In Appendix we prove the exponential growth of the Cauchy solutions of the one-dimensional difference and differential equations with random weakly correlated coefficients. This is an essential point of the proof of the absence of the absolutely continuous component in the one-dimensional operator spectrum and even of the purely point character of the spectrum (see [7]).

\section{General Spectral Properties of Infinite Disordered Systems}

To simplify our discussion, we consider as a typical but fairly simple object, the multidimensional Jacobi matrices with random diagonal elements, but all our statements as well as the essence of their proofs are valid also in much more general situations.

Let $l_{2}\left(\mathbb{Z}^{d}\right)$ be the space of sequence $\left\{x_{k}\right\}, \sum_{k \in \mathbb{R}^{d}}\left|x_{k}\right|^{2}<\infty$ on the periodic $d$-dimensional lattice $\mathbb{Z}^{d}=\left\{\mathrm{k}: \sum_{1}^{d} \mathrm{k}_{\alpha} \mathrm{a}_{\alpha}, \mathrm{k}_{\alpha} \in \mathbb{Z}^{1} a_{\alpha}\right.$ are certain fixed linearly independent vectors of $\left.R^{d}\right\}$ and $H(\omega)$ be the family of bounded operators of the form:

$$
(H x)_{k}=\sum_{\delta} x_{k+\delta}+v_{k}(\omega) x_{k} .
$$

Here $k+\delta$ are the vectors corresponding to the nearest neighbours of $k, \mathrm{v}_{k}(\omega)$ is the real homogeneous metrically transitive bounded sequence:

$$
\left|v_{k}(\omega)\right| \leqq C<\infty
$$

1 This property of the physical quantities of large disordered systems will be called self-averaging (the term was introduced by I. M. Lifshitz)

2 The main ideas and results of this paper were first published in [6] 
and $\omega$ is a point of the realization space $\Omega$. As is well known [5], every homogeneous metrically transitive sequence defines in its realization space $\Omega$ the probability preserving group of the transformation $T_{l}$ satisfying the relation

$$
v_{k+l}(\omega)=v_{k}\left(T_{l} \omega\right)
$$

This group of the transformations has no nontrivial invariant subspaces in $\Omega$ [5]. Such properties of random quantities $v_{k}(\omega)$ give rise to the relation

$$
H\left(T_{l} \omega\right)=U_{l} H(\omega) U_{-l},
$$

where $U_{i}$ is the unitary group of shift operators in $l_{2}\left(\mathbb{Z}^{d}\right)$

$$
\left(U_{l} x\right)_{k}=x_{k+l} .
$$

The matrix form of Eq. (3) is

$$
H_{k, q}\left(T_{l} \omega\right)=H_{k+l, q+l}(\omega) .
$$

It is clear that if Eq. (3) holds for the family $\{H(\omega)\}$ it also holds for any function of $H$, in particular for $e^{i t H}$ and for $E(\lambda)$ where $E(\lambda)$ is the spectral family (resolution of identity) [8] of the operator $H$.

Henceforth the family of the bounded operators $\{H(\omega)\}$ in $l_{2}\left(\mathbb{Z}^{d}\right)$ will be called metrically transitive (ergodic) if for almost all $\omega \in \Omega$ they satisfy Eq. (3) and if quantity $(H x, y)$ is a measurable function of $\omega$ for $x$ and $y$ belonging to a certain dense set in $l_{2}\left(\mathbb{Z}^{d}\right)^{3}$.

If $\Omega$ consists of a single point, then $H$ is the difference operator with constant coefficients, i.e. it is a particular case of the Toeplitz operator [9]. It is convenient to consider further examples of our objects, which are more complicated, in the continuous case (the Schrödinger equation in $R^{d}$ with random coefficients), with restricting oneself, only for the sake of simplicity, to dimension 1 .

Example 1. Let $\Omega$ be a circle of radius $R, P(\cdot)$ the Lebesgue measure on this circle, divided by $(2 \pi R)^{-1}, T_{x}$ rotation by an angle $x$. Let $v(x, \omega)=v(x+\omega)$ where $v(x)$ is a $2 \pi R$-periodic function. In other words, $\{v(x, \omega)\}$ is the set of $2 \pi R$-periodic functions with random origin $\omega$ uniformly distributed over a fixed interval of length $2 \pi R$.

Example 2. Let $\Omega$ be an $n$-dimensional torus with incommensurable radii $R_{1}, \ldots, R_{n}, P(\cdot)$ the normalized Lebesgue measure on it, $T_{x} \omega=\left\{\omega_{i}+x, \bmod 2 \pi R_{i}\right.$, $i=1, \ldots, n\}, v(x, \omega)=F\left(T_{x} \omega\right)$ where $F(\omega)$ is a function on the torus. Such $v(x)$ are called quasiperiodic functions [10] (the spectrum of such operators was studied in [25] and in [27]).

Therefore all our results may be applied, e.g., to the Schrödinger equation in $R^{d}$ with a periodic, quasiperiodic and even general almost periodic $(n=\infty)$ potential.

3 If operators $H(\omega)$ are unbounded for almost all $\omega \in \Omega$, it should be required that almost all $H(\omega)$ have the same domain $D$ which is invariant with respect to $U_{l}: U_{l} D \subset \mathscr{D}$ for all $l \in \mathbb{Z}^{d}$ 
Proposition 1. Let $P(\omega)$ be a random ergodic operator which is an orthogonal projection for almost every $\omega$ and let $v$ be its dimension: $v=\operatorname{Tr} P$. Then either $v=0(P \equiv 0)$ or $v=\infty$ with probability 1 . The former possibility is realized if and only if $E\left\{P_{k k}\right\}=0^{4}$, and the latter when $E\left\{P_{k k}\right\}>0$. Here $P_{k l}$ is matrix elements of $P$.

Proof. It follows from Eqs. (3) or (4) for $P(\omega)$ that

$$
v(\omega)=\sum_{k \in \mathbb{Z} d} P_{k k}(\omega)
$$

is the invariant function on $\Omega: v\left(T_{l} \omega\right) \leqq v(\omega)$. Since $T_{l}$ is ergodic, $v$ with probability 1 is independent of $\omega$ and $E\left\{P_{k k}\right\}$ is independent of $k$ [see Eq. (4)]. Thus, for an arbitrary integer

$$
v=E\{v\} \geqq \sum_{\left|k_{\alpha}\right| \leqq n} E\left\{P_{k k}\right\}=(2 n+1)^{d} E\left\{P_{00}\right\}
$$

and if $v<\infty$, then $E\left\{P_{00}\right\}=0$ and then by Eq. (5), $v=0$. If $E\left\{P_{00}\right\}>0$, then $v=\infty$.

Applying Proposition 1 to the projection operator $E(\Delta)=E\left(\lambda_{2}\right)-E\left(\lambda_{1}\right),\left(\lambda_{1}, \lambda_{2}\right]$ being a semiclosed interval, we obtain the following theorem:

Theorem 1. Let $\Delta$ be any fixed semiclosed interval on the spectral axis. Then with probability 1 , the number of spectrum points of $H(\omega)$ belonging to $\Delta$ equals zero or infinity. Which of these possibilities is realized, depends on whether $E\left\{E_{k k}\right\}$ is zero or not.

Let $\Sigma(\omega)$ be the spectrum of the selfadjoint operator $H(\omega)$, i.e. the set of growth points of the spectral family $E(\lambda)$. Theorem 1 shows that with probability 1 the spectrum of $H(\omega)$ is essential [8], i.e. every point of $\Sigma(\omega)$ is its limit point.

Theorem 2. Every point $\lambda$ on the spectral axis either belongs to the spectrum of almost all operators $H(\omega)$, or does not belong to almost any of them. The realization of one of these possibilities depends on whether $\lambda$ is or is not the growth point of the non-decreasing function $E\left\{E_{00}(\lambda)\right\}$.

Proof. The first part of the theorem follows from relation

$$
\{\omega: \lambda \in \Sigma(\omega)\}=\bigcap_{\varepsilon>0}\{\omega:(\lambda-\varepsilon, \lambda+\varepsilon) \cap \Sigma(\omega) \neq 0\}
$$

and from Theorem 1, since every event in the right-hand side has the probability 0 or 1 only.

Let us prove the second part of the theorem. If $\lambda$ is a point of the growth of $E\left\{E_{00}(\lambda)\right\}$, i.e. for every $\varepsilon>0$

$$
E\left\{E_{00}(\lambda+\varepsilon)-E_{00}(\lambda-\varepsilon)\right\}>0
$$

then with nonzero probability,

$$
E_{00}(\lambda+\varepsilon)-E_{00}(\lambda-\varepsilon)>0
$$

i.e. the interval $(\lambda-\varepsilon, \lambda+\varepsilon)$ contains the points of $\Sigma(\omega)$. Since the probability of this event is nonzero, then, as specified by Theorem 1, it equals 1 . Then, with

4 The symbol $E\{\ldots\}$ denotes the expectation with respect to the probability measure $P(\cdot)$ 
probability 1 , the points of $\Sigma(\omega)$ are contained in $(\lambda-\varepsilon, \lambda+\varepsilon)$ for every $\varepsilon>0$. Since $\Sigma(\omega)$ is a closed set, $\lambda$, with probability 1 , is contained in $\Sigma(\omega)$ too.

Now let $\lambda$ be in $\Sigma(\omega)$ with probability 1 and let $\lambda$ be not a growth point of $E\left\{E_{00}(\lambda)\right\}$, i.e. for some $\varepsilon>0$,

$$
E\left\{E_{00}(\lambda+\varepsilon)-E_{00}(\lambda-\varepsilon)\right\}=0 .
$$

In accordance with Theorem 1, this equation implies that the interval $(\lambda-\varepsilon, \lambda+\varepsilon)$ does not contain, with probability 1 , the points of the spectrum of operators $H(\omega)$.

Corollary 1. The spectra of almost all $H(\omega)$ coincide, i.e. $\Sigma$ is a nonrandom set.

The proof of the corollary immediately follows from the fact that every $\Sigma(\omega)$ is the set of growth points of the nonrandom function $E\left\{E_{00}(\lambda)\right\}$. Another way of proving this corollary is to use Eq. (2) directly.

Let us show now that for the point spectrum of $H(\omega)$ [it is the set of the discontinuity points of $E(\lambda)]$, only the second possibility of Theorem 2 is realized.

Theorem 3. The event: any fixed point $\lambda$ is the eigenvalue of finite multiplicity has the zero probability.

Proof. If $\lambda$ lies in the point spectrum $\Sigma_{P}$ of $H$, then the projection operator $P(\lambda)$ $=E(\lambda+0)-E(\lambda)^{5}$ is nonzero. Since $P(\lambda)$ is the strong limit for $\varepsilon \downarrow 0$ of the projection operators $E(\lambda+\varepsilon)-E(\lambda-\varepsilon)$ it satisfies Eq. (2) for every fixed $\lambda$, i.e. $P(\lambda)$ is a metrically transitive (ergodic) projection operator. Then by Proposition $1, \operatorname{dim} P(\lambda)$ $=0, \infty$ only. Since, by supposition, $\operatorname{dim} P(\lambda)<\infty$ with probability $1, P(\lambda)=0$.

One may think that from this Theorem it follows that $H$ has no point spectrum, but it is not so. The point spectrum is just too "mobile" and "sensitive" to the change of the potential set, and therefore this set varies with $\omega$. Besides, since it is countable, the probability for some fixed point to fall into this rather fine and mobile set is zero. Loosely speaking, Theorem 3 means that the point spectrum of $H(\omega)$ has continuous probability distribution.

However, if one considers the intervals instead of the points, it turns out that the point spectrum $\Sigma_{P}(\omega)$ has the property similar to that of the whole spectrum $\Sigma(\omega)$. In order to prove this property, let us introduce quantity

$$
A_{k q}(\Delta, \omega)=\sum_{\lambda \in \Delta \cap \Sigma_{P}}\left|P_{k q}(\lambda, \omega)\right|^{2},
$$

where $P(\lambda)=s$ - $\lim _{\varepsilon \downarrow 0}[E(\lambda+\varepsilon)-E(\lambda-\varepsilon)]$. Since $P_{k q}(\lambda)$ are the jumps of the bounded variation function $E_{k q}(\lambda)$ then by the Wiener theorem ${ }^{6}$ we find that

$$
A_{k q}=\lim _{\tau \rightarrow \infty} \tau^{-1} \int_{0}^{\tau}\left|B_{k q}(t)\right|^{2} d t
$$

5 As is usual in spectral theory of linear operators [8], resolution of identity $E(\lambda)$ is the leftcontinuous function

6 This theorem is that if $\sigma(\lambda)$ is a bounded variation function, $\delta_{i}$ are its jumps and $m(t)$ is its Fourier transform, then

$$
\lim _{\tau \rightarrow \infty} \tau^{-1} \int_{0}^{\tau}|m(t)|^{2} d t=\sum_{i}\left|\delta_{i}\right|^{2}
$$


where

$$
B_{k q}(t)=\int_{\Delta} e^{i \lambda t} d E_{k q}(\lambda)
$$

or $B_{k q}(t)$ are the matrix elements of the operator $e^{i t H} E(\Delta)$. Thus, $A_{k q}$ satisfy Eq. (4), i.e.,

$$
A_{k q}\left(\Delta, T_{l} \omega\right)=A_{k+l, q+l}(\Delta, \omega) .
$$

Theorem 4. With probability 1 the number of eigenvalues in the fixed interval $\Delta$ is zero or infinity. The first case is realized if and only if $E\left\{A_{00}(\Delta)\right\}=0$ and the second one if and only if $E\left\{A_{00}(\Delta)\right\}>0$.

Proof. Let

$$
\mu(\Delta, \omega)=\sum_{k, q \in \mathbb{Z}^{d}} A_{k q}(\Delta, \omega) .
$$

By Eq. (6),

$$
\mu(\Delta)=\sum_{\lambda \in \Delta \cap \Sigma_{P}} \operatorname{dim} P(\lambda)
$$

i.e. $\mu(\Delta)$ is the number of eigenvalues in the interval $\Delta$ (with allowance for their multiplicty). According to Eqs. (7) and (8), $\mu\left(\Delta, T_{l}(\omega)=\mu(\Delta, \omega)\right.$ and owing to metrical transitivity of the group $T_{l}$ it is independent of $\omega$. Further reasoning is the same as in the proof of Proposition 1 and uses relation $E^{2}\left\{A_{k q}\right\} \leqq E\left\{A_{k k}\right\} \cdot E\left\{A_{q q}\right\}$.

The proved theorem shows, in particular, that the point spectrum, if any, has no isolated points of finite multiplicity (discrete eigenvalues) with probability 1, i.e. it is a dense countable set in which every point is a limit one.

Quantity $A_{00}(-\infty, \infty)$ was introduced in [11] where its positivity served as a criterion of absence of "quantum" diffusion of the particles. Indeed, as $A_{00}(-\infty, \infty)$ is the quantum mechanical probability for a particle which is at site 0 at $t=0$ to return to this site after an infinite time, it is clear that if $A_{00}>0$ the initial wave packet does not spread over all the volume of the macroscopically large system but remains mainly localized in the vicinity of its initial position. It is widely agreed that in such a case the states are localized. In the opposite case, where the wave packet is gradually spreading over the entire space and therefore disappearing from any finite domain, the states should be delocalized (extended).

As we have seen, the absence (or presence) of such diffusion in the neighbourhood of some $\lambda$ is equivalent to the presence (or absence) of the point spectrum in the neighbourhood of this $\lambda$.

There is also another criterion of localization of the eigenfunctions. In order to explain it, let us write the Kubo-Greenwood formula for the active conductivity of free electron gas in the random (impurity) field. According to $[12]\left(\hbar^{2}=2 m=1\right)$.

$$
\sigma_{\alpha \beta}(\omega)=4 \pi e^{2} \lim _{\varepsilon \downarrow 0} \lim _{V \rightarrow \infty} \int_{-\infty}^{\infty} \frac{n(\lambda)-n\left(\lambda^{\prime}\right)}{\lambda^{\prime}-\lambda} \cdot \frac{\varepsilon}{\varepsilon^{2}+\left(\lambda-\lambda^{\prime}+\omega\right)^{2}} F_{V}^{\alpha \beta}\left(d \lambda, d \lambda^{\prime}\right),
$$


where $n(\lambda)=\left(\exp -\lambda-E_{F} / T-1\right)^{-1}$ is the Fermi distribution function, $E_{F}$ is the Fermi energy (see Theorem 13 below),

$$
\begin{aligned}
F_{V}^{\alpha \beta}\left(\Delta, \Delta^{\prime}\right) & =\frac{1}{V} \sum_{\substack{\lambda_{n} \in \Delta \\
\lambda_{m} \in \Delta^{\prime}}} u_{m n}^{\alpha} u_{n m}^{\beta}{ }^{7} \\
u_{n m}^{\alpha} & =\int_{V} \psi_{n}^{*}(\mathbf{r}) \partial \psi_{m} / \partial r_{\alpha} \mathbf{d r}
\end{aligned}
$$

and $\lambda_{n}$ and $\psi_{n}(\mathbf{r})$ are energy levels and states of the finite system enclosed in the volume $V$.

According to [1], the states in the neighbourhood of a given $\lambda$ are localized, if the static conductivity $\sigma_{\alpha \beta}(0)$ for $T=0$ and $\lambda=E_{F}$ is zero, and are delocalized in the opposite case.

It is clear that the behaviour of $\sigma_{\alpha \beta}(\omega)$ for $\omega \rightarrow 0$ is determined by the behaviour of the limiting measure $F^{\alpha \beta}\left(\Delta, \Delta^{\prime}\right)$ on the diagonal in the $\left(\lambda, \lambda^{\prime}\right)$ plane. Using the same line of reasoning as in the proof of the Loomis theorem $[13]^{8}$, one can prove

\section{Theorem 5}

$$
\sigma_{\alpha \beta}(0)=\lim _{\omega \downarrow 0} \sigma_{\alpha \beta}(\omega)=\int_{-\infty}^{\infty} \frac{\partial n(\lambda)}{\partial \lambda} d \Phi_{\alpha \beta}(\lambda)
$$

where

$$
\Phi_{\alpha \beta}(\Delta)=\lim _{\omega \downarrow 0} \lim _{\delta \downarrow 0} \frac{1}{2 \delta} F^{\alpha \beta}\left\{\left(\lambda, \lambda^{\prime}\right): \lambda, \lambda^{\prime} \in \Delta,\left|\lambda-\lambda^{\prime}+\omega\right| \leqq \delta\right\}
$$

and if there exists the limit in one side of Eq. (10), then there is one in the other side too.

If the measure $F^{\alpha \beta}\left(\Delta, \Delta^{\prime}\right)$ is absolutely continuous with respect to the Lebesgue measure,

$$
F^{\alpha \beta}\left(\Delta, \Delta^{\prime}\right)=\int_{\Delta \times \Delta^{\prime}} f_{\alpha \beta}\left(\lambda, \lambda^{\prime}\right) d \lambda d \lambda^{\prime}
$$

then

$$
\Phi_{\alpha \beta}(\Delta)=\int f_{\alpha \beta}(\lambda, \lambda) d \lambda
$$

7 As is shown in [4], $F_{V}^{\alpha \beta}\left(\Delta, \Delta^{\prime}\right)$ is a selfaveraging quantity, i.e. it tends with probability 1 to nonrandom limit as $V \rightarrow \infty$ [see Eq. (17) below]

8 This theorem asserts that, if $f(z)=\frac{1}{\pi} \int \frac{d \tau(\lambda)}{\lambda-z} . \operatorname{Im} z \neq 0, \tau(\lambda)$ is a bounded nondecreasing function, then $\lim _{\varepsilon \downarrow 0} \operatorname{Im} f(\lambda+i \varepsilon)=\tau^{(-)}(\lambda)$ where $\tau^{(-)}(\lambda)=\frac{\lim \tau(\lambda+\varepsilon)-\tau(\lambda-\varepsilon)}{2 \varepsilon}$ is symmetrical derivative of $\tau(\lambda)$ and the existence of the limit in one side suggests existence of the limit in the other side 
i.e. $\sigma_{\alpha \beta}(0)$ is indeed determined by the behaviour of the density $f_{\alpha \beta}\left(\lambda, \lambda^{\prime}\right)$ near the line $\lambda=\lambda^{\prime}$ in $\left(\lambda, \lambda^{\prime}\right)$ plane. For a free particle $(v(x)=0)$.

$$
\begin{aligned}
& f_{\alpha \beta}\left(\lambda, \lambda^{\prime}\right)=\delta_{\alpha \beta} \lambda \delta\left(\lambda-\lambda^{\prime}\right) \varrho_{d}(\lambda), \lambda, \lambda^{\prime} \geqq 0 \\
& \varrho_{d}(\lambda)=C_{d} \lambda^{d / 2-1}, \quad C_{d}=(4 \pi)^{-d / 2} \Gamma^{-1}\left(\frac{d}{2}-1\right)
\end{aligned}
$$

and corresponding static conductivity equals infinity. In the disordered case it is widely believed that $\left.\sigma_{\alpha \beta}(0)\right|_{T=0} \equiv \sigma_{\alpha \beta}\left(E_{F}\right)=0$ in the one-dimensional case 9 . But in the three-dimensional case, $\sigma_{\alpha \beta}\left(E_{F}\right)$ is zero only when $E_{F}$ lies in the low energy end of the band. The boundary value $E_{c}$ of energy such that $\sigma_{\alpha \beta}\left(E_{F}\right)=0$ for $E_{F}<E_{c}$ and $\sigma_{\alpha \beta}\left(E_{F}\right) \neq 0$ for $E_{F}>E_{c}$ is called the mobility edge [2].

It was assumed in [1-3] that $E_{c}$ is at the same time the boundary between localized and delocalized states. As follows from Theorem 4, in such situation the equality

$$
E\left\{A_{00}(\Delta)\right\}=0, \quad \Delta \in\left(E_{c}, \infty\right)
$$

must hold ${ }^{10}$.

These assumptions imply implicitly impossibility of coexistence of localized and delocalized states for the same energy, however for slowly decreasing and fastly oscillating potentials, such coexistence is feasilbe in terms of the operator theory. Yet, such coexistence is usually the result of very subtle interference phenomena, and therefore such states must be unstable and should disappear in response to even slight changes of the potential. Otherwise speaking, for a randomchosen potential $v(x)$ this situation can hardly be the case.

It is of some interest that the calculation of both $E\left\{A_{k q}\right\}$ and $\sigma_{\alpha \beta}\left(E_{F}\right)$ is reduced to calculation of the measures of the diagonal in the $\left(\lambda, \lambda^{\prime}\right)$ plane:

$$
\mathfrak{U}_{k q}\left(\Delta, \Delta^{\prime}\right)=E\left\{\int_{\Delta \times \Delta^{\prime}} d E_{k q}(\lambda) d E_{q k}\left(\lambda^{\prime}\right)\right\}
$$

in case of $A_{k q}(\Delta)$ and

$$
F^{\alpha \beta}\left(\Delta, \Delta^{\prime}\right)=E\left\{\left.\int_{R^{d}} \frac{\partial E\left(\mathbf{r}, \mathbf{r}^{\prime}, \Delta\right)}{\partial r_{\alpha}} \cdot \frac{\partial E\left(\mathbf{r}^{\prime}, \mathbf{r}, \Delta^{\prime}\right)}{\partial r_{\beta}^{\prime}}\right|_{r=0} d \mathbf{r}^{\prime}\right\}
$$

in the case of conductivity. But in the former case the density of $\mathfrak{A}_{k q}$ should have the singularity of the form $\delta\left(\lambda-\lambda^{\prime}\right) a_{k q}(\lambda)$ with $a_{k q}>0$ for $\lambda<E_{c}$ (in the ordered case $a_{k q}(\lambda)=0$ for all $\left.\lambda\right)$ and in the latter case the density of $F^{\alpha \beta}\left(\lambda, \lambda^{\prime}\right)\left[\right.$ i.e. $f_{\alpha \beta}\left(\lambda, \lambda^{\prime}\right)$ of Eq. (11)] equals zero for $\lambda=\lambda^{\prime}, \lambda<E_{c}$ (in the ordered case, as we have seen, it has a singularity of $\delta\left(\lambda-\lambda^{\prime}\right)$ type on the diagonal).

In conclusion of this section we shall formulate, without a proof, a theorem on the unbounded ergodic operators, supposing that all $H(\omega)$ have the same domain $D[8]$ with the property $U_{l} D \subset D$ for all $l \in \mathbb{Z}^{d}$.

9 This fact was proved recently by H. Kunz and B. Souillard for the case of independent $v_{k}$ [26]

10 We assume that $A_{00}(-\infty, \lambda)$ is left-continuous function of $\lambda$ 
Theorem 6. A symmetric ergodic operator $H(\omega)$ has, with probability 1 , zero deficiency indices, or with the same probability, infinite deficiency indices ${ }^{11}$.

\section{One-Dimensional Disordered Systems}

In this Section we shall consider the operator $(1)$ in $l_{2}(-\infty, \infty)$ [or the Schrödinger equation in $\left.\mathscr{L}_{2}\left(R^{1}\right)\right]$. We begin by stating two theorems which are straightforward corollaries of Theorems 6 and 3 of the preceding section.

Theorem 7. The operator $H(\omega)$ is essentially selfadjoint with probability 1 in the onedimensional case.

Theorem 8. The probability for any fixed point $\lambda$ to be an eigenvalue of the onedimensional operator (1) is zero.

Let us consider now the continuous spectrum $\Sigma_{c}(\omega)$ of $H(\omega)$, i.e. the set of nonisolated growth points of the spectral family $E(\lambda)[8]$. Rather an important part of $\Sigma_{c}$ is the absolutely continuous spectrum $\Sigma_{a c}$. It is the spectrum of $H_{a c}$ where

$$
H_{a c}=\left.H\right|_{l_{a c}} .
$$

$l_{a c}=\left\{x: x \in l_{2}(-\infty, \infty),(E(\lambda) x, x)\right.$ is absolutely continuous with respect to the Lebesgue measure $\}$. It is known [14] that $\Sigma_{a c}=\Sigma$ in the ordered case. We shall prove now that one-dimensional disordered systems have no absolutely continuous part of the spectrum: $\Sigma_{a c}=\phi$. This was proved first in [15] and later in [16] and [6] (see below). All the proofs were essentially based on a certain property of the solution $u_{n}(\lambda)$ of the Cauchy problem for Eq. (1), i.e.

$$
u_{n+1}+u_{n-1}+v_{n} u_{n}=\lambda u_{n}, \quad u_{-1}=\cos \varphi, \quad u_{0}=\sin \varphi .
$$

Namely, we assume that for fixed $\lambda$ and $\varphi$ the limits

$$
\lim _{n \rightarrow \pm \infty} \frac{1}{|n|} \ln \left(u_{n}^{2}+u_{n-1}^{2}\right)=\mathfrak{J}_{ \pm}(\lambda)
$$

exist with probability 1 , are independent of $\varphi$ and $\omega$ and are strictly positive for this $\lambda$. If this assumption holds, we call the solution of the Cauchy problem (13) an exponentially increasing solution with probability 1 for this $\lambda$. At first sight, such property of $u_{n}(\lambda)$ from (13) seems rather unusual. However it appears to be even typical for metrically transitive $v_{n}$ with sufficiently fast decay of statistical correlations between $v_{n}$ and $v_{m}$ as $|n-m| \rightarrow \infty$. This question will be discussed in more detail at the end of this section (Proposition 2) and in Appendix.

Theorem 9. Let $v_{n}$ be such a metrically transitive sequence that the corresponding Cauchy solutions for all $\lambda$ increase exponentially at least at one of the ends of the

11 This theorem was proved by A. L. Figotin and the present author (unpublished) 
interval $(-\infty, \infty)$. Then with probability 1 , the spectrum of $H(\omega)$ has no absolutely continuous component.

Proof. As is known in the spectral theory of difference operators [17], the spectral function $\varrho(\lambda)$ plays an important role since it determines the Parseval identity and is, in a way, a derivative of resolution of identity $E(\lambda)$. The spectral function $\varrho(\lambda)$ is characterized by the following properties:

a) for any $x \in l_{2}(-\infty, \infty)$ the nondecreasing function $(E(\lambda), x, x)$ is absolutely continuous with respect to $\varrho(\lambda)$ and hence if the spectrum of $H$ has an absolutely continuous part, then $\varrho(\lambda)$ has a component absolutely continuous with respect to the Lebesgue measure;

b) for $Q$-almost every $\lambda$, the Cauchy solutions $u_{\alpha, n}(\lambda) \alpha= \pm 1$ of (13) corresponding to $\varphi=0, \frac{\pi}{2}$ satisfy inequality ${ }^{12}$ :

$$
\left|u_{\alpha, n}(\lambda)\right| \leqq C(\lambda, \varepsilon)|n|^{1 / 2+\varepsilon}, \quad \varepsilon>0
$$

Assume now that for some $\omega$ the spectrum of $H(\omega)$ has an absolutely continuous component. Then the set of $\lambda$ satisfying (15) for a given $\omega$ has the positive Lebesgue measure. We shall see now that this fact contradicts the property of exponential growth. Indeed, let $\tilde{P}$ be the measure on the set of pairs $(\omega, \lambda), \omega \in \Omega \lambda \in R^{1}$, which is equal to the product of probability measure $P$ on $\Omega$ and the Lebesgue measure on the spectral axis. It is clear that $u_{n}^{2}(\lambda)+u_{n+1}^{2}(\lambda)$ is a $\tilde{P}$-measurable function of $(\omega, \lambda)$. Then, by our assumption, the set of pairs $(\omega, \lambda)$, for which Eq. (14) holds, has the full $\tilde{P}$-measure. Then its complement has zero $\tilde{P}$-measure, whence by the Fubini theorem, for almost every $\omega \in \Omega$, the Lebesgue measure of the set of $\lambda$ for which (14) does not hold, is equal to zero as well. But this set contains all the points $\lambda$ for which Eq. (15) is true, and therefore the set of such $\lambda$ cannot have a positive Lebesgue measure, in contradiction with our assumption.

Our arguments in fact prove a stronger statement, such that Theorems 3,8 and 9 are simple corollaries to it:

Theorem 10. Under the conditions of Theorem 9, with probability 1, the measure which is determined by spectral function $\varrho(\lambda)$ is singular (disjoint) with respect to any fixed (nonrandom) measure $F(\lambda)$ on $R^{1}$.

Loosely speaking, this theorem asserts that the probability of coincidence of $\varrho(\lambda)$ with any fixed nondecreasing function $F(\lambda)$ is zero.

The existence of such generalization of Theorem 9 was suggested by Y. G. Sinai and proved by the author of this paper under some additional assumptions. I. Goldsheid pointed out that the proof of Theorem 10 in fact repeats that of Theorem 9.

Now discuss some cases of exponential growth of the Cauchy solutions of (13). According to [18], for every fixed $\lambda \in R^{1}$ and $\varphi \in\left(-\frac{\pi}{2}, \frac{\pi}{2}\right]$ there exist with probabili-

12 This inequality can be obtained by taking it into account that the generalized eigenfunctions of $H$ are elements of $l_{2}(-\infty, \infty)$ with the weight $|n|^{-(1+2 \varepsilon)}, \varepsilon>0$, i.e. the extension of the usual $l_{2}$ for which $\sum_{n}\left|x_{n}\right|^{2}|n|^{-(1+2 \varepsilon)}<\infty[17]$ 
ty 1 both the limits:

$$
\lim _{n \rightarrow \pm \infty} \frac{1}{|n|} \ln \left(u_{n}^{2}+u_{n+1}^{2}\right) \equiv \mathfrak{J}_{ \pm}(\lambda, \omega, \varphi)
$$

and each of them as a function of $\varphi$ takes on two nonrandom values $\pm \mathfrak{I}_{ \pm}(\lambda)$ $\left(\mathfrak{J}_{ \pm}(\lambda) \geqq 0\right)$ in the case of metrically transitive $v_{n}$. Let

$$
\left.\Omega(\lambda, \varphi) \equiv\left\{\omega: \mathfrak{I}_{+}(\lambda, \omega, \varphi)=\mathfrak{I}_{+}\right)\right\} \cup\left\{\omega: \mathfrak{I}_{-}(\lambda, \omega, \varphi)=\mathfrak{I}_{-}(\lambda)\right\}
$$

i.e. $\Omega(\lambda, \varphi)$ consists of such $\omega$ for which the Cauchy solutions have exponential growth at least at one of the ends of the interval $(-\infty, \infty)$. The complement $\Omega^{\prime}$ of $\Omega(\lambda, \varphi)$ consists of $\omega$ for which the solution of $(13)$ exponentially decays at both the ends of $(-\infty, \infty)$. In this case the correspondent $\lambda$ is an eigenvalue of $H$. By Theorem $3, P\left\{\Omega^{\prime}\right\}=0$, so that $P\{\Omega(\lambda, \varphi)\}=1$ for every $\lambda$ and $\varphi$. We see now that, if $\mathfrak{J}_{ \pm}(\lambda)>0$, for $\lambda \in \Sigma$, then the condition of Theorem 9 is fulfilled. In Appendix we prove

Proposition 2. If $v_{n}$ is an ergodic and invertible Markov chain (for definitions, see Appendix), then the quantities $\mathfrak{I}_{ \pm}(\lambda)$ are strictly positive for all $\lambda$.

Thus we have

Theorem 11. If the random sequence $v_{n}$ satisfies the conditions of Proposition 2, then the operators $H(\omega)$ with probability 1 have no absolutely continuous spectrum.

In particular, $\mathfrak{J}_{ \pm}(\lambda)>0$, for statistically independent $v_{n}$. In this important case, the exponential increase of the Cauchy solutions was proved in [19], using results of [20] for the asymptotic behaviour of the random matrices products. Positiveness of $\mathfrak{J}_{ \pm}(\lambda)$ is also essential to prove that the one-dimensional operator $H(\omega)$ (and its continuous analogue, the Schrödinger equation) have with probability 1 a pure point spectrum $\left(\Sigma=\Sigma_{P}\right)$ if $v_{n}$ is a sufficiently smooth Markov chain [7].

\section{Asymptotic Properties of Large Finite Disordered Systems}

Till now we have dealt with the operators $H(\omega)$ determined by operation (1) on the whole lattice $\mathbb{Z}^{d}$. Let us consider now the $(2 n+1)^{d}$ dimensional space $l_{2}\left(C_{n}\right)$ of sequences

$$
x \in C_{n}=\left\{\left\{x_{k}\right\}: \sum_{1}^{d} k_{\alpha} a_{\alpha}=k,\left|k_{\alpha}\right| \leqq n\right\}
$$

and the operator $H_{n}(\omega)$ in it:

$$
\left(H_{n}\right)_{k q}=\left\{\begin{array}{l}
H_{k q},\left|q_{\alpha}\right|,\left|k_{\alpha}\right| \leqq n, \forall_{\alpha} \\
0,\left|q_{\alpha}\right|,\left|k_{\alpha}\right|>n \text { for some } \alpha .
\end{array}\right.
$$


Let $E_{n}(\lambda)$ be the resolution of identity of $H_{n}$, and

$$
N_{n}(\lambda)=Q^{-1} \operatorname{Tr} E_{n}(\lambda)=Q^{-1} \sum_{\lambda_{i}(n)<\lambda} 1,
$$

where $\lambda_{1}(n) \leqq \ldots \leqq \lambda_{Q}(n), Q=(2 n+1)^{d}$ are eigenvalues of $H_{n}$.

Theorem 12 [4]. Let $v_{n}$ be a homogeneous and ergodic random sequence satisfying (2). Then there exists such a nonrandom nondecreasing function $N(\lambda)$ that with probability 1

$$
\lim _{n \rightarrow \infty} N_{n}(\lambda)=N(\lambda)
$$

at every continuity point of $N(\lambda)$ and

$$
N(\lambda)=E\left\{E_{00}(\lambda)\right\} \text {. }
$$

If $\Omega=\{0\}$ (ordered case), $H$ is a Toeplitz operator and Eq. (16) is a particular case of rather general theorem of Szegö [9].

The function $N(\lambda)\left[N^{\prime}(\lambda)\right.$ is called the density of states of operator $\left.H\right]$ can be calculated in many cases, especially in one-dimensional situations (see, e.g. [21, 22]).

A similar theorem holds for more complex quantities constructed of eigenelements of $H[4,23]$. In particular, equality

$$
\lim _{V \rightarrow \infty} F_{V}^{\alpha \beta}\left(\lambda, \lambda^{\prime}\right)=E\left\{\left(p_{\alpha} E(\lambda) p_{\beta} E\left(\lambda^{\prime}\right)\right)(0,0)\right\}
$$

is true with probability 1 for function $F_{V}^{\alpha \beta}\left(\lambda, \lambda^{\prime}\right)$ of $(9)$, where $\mathbf{p}=-\nabla$ is the momentum operator, and $E(\lambda, x, y)$ is the kernel of operator $E(\lambda)$.

Theorems 12 and 2 imply

Theorem 2'. The point $\lambda$ belongs or does not belong to the spectrum $\Sigma$ of $H$ with probability 1. The realization of either possibility depends on whether or not $\lambda$ is a growth point of $N(\lambda)$.

Theorem 13. If $\operatorname{dim} P(\lambda)<\infty$, for all $\lambda \in \Sigma$ for almost every $\omega$, then function $N(\lambda)$ is continuous.

The proof follows from the relation

$$
N(\lambda+0)-N(\lambda)=E\left\{P_{00}(\lambda)\right\},
$$

the Proposition 1 and the left continuity of $N(\lambda)$.

Corollary 2. The function $N(\lambda)$ corresponding to the one-dimensional difference and differential operators is continuous.

Theorem 2 and Corollary 2 in somewhat different formulation, for operator (1) in $l_{2}(0, \infty)$ were proved in $[15,16]$. The authors of $[15,16]$ concluded from these two conditions that the spectrum of $H$ cannot contain isolated points. In our scheme this fact is the direct corollary of Theorem 1 which, in turn, is a simple consequence of the properties of spatial homogeneity and decay of correlation. 
Theorem 14. Let $\lambda_{1}(n) \leqq \ldots \leqq \lambda_{Q}(n)$ be the eigenvalues of $H_{n}$. Then with probability 1 there exists the limit

$$
\lim _{n \rightarrow \infty, k / n \rightarrow \infty} n^{-1}\left(\lambda_{1}(n)+\ldots+\lambda_{k}(n)\right) \equiv E_{F}(\varrho),
$$

where

$$
E_{F}(\varrho)=\varrho^{-1} \int_{0}^{\varrho} L(\tau) d \tau
$$

and

$$
L(\tau)=\inf \{\lambda: N(\lambda) \geqq \tau\}
$$

is the inverse function to $N(\lambda) . E_{F}(\varrho)$ is the Fermi energy of the ideal (noninteracting) Fermi gas in the random field.

Proof. By Theorem 12 and Lemma 3 from [24], the sequence $L_{n}(\tau)=\inf \left\{\lambda: N_{n}(\lambda)\right.$ $\geqq \tau, 0 \leqq \tau \leqq 1\}$ converge with probability 1 to $L(\tau)$ in the $\mathscr{L}_{1}(0,1)$ metric, i.e.

$$
\lim _{n \rightarrow \infty} \int_{0}^{1}\left|L_{n}(\tau)-L(\tau)\right| d \tau=0
$$

with probability 1 . Now Theorem 14 follows from (18) and from relation

$$
Q^{-1} \sum_{i=1}^{k} \lambda_{i}(n)=\frac{k}{Q} \int_{0}^{k / Q} L_{n}(\tau) d \tau .
$$

This theorem was first proved by Shubin (see, e.g., [23]).

In conclusion we shall show that the analogue of Theorem 10 in the space $l_{2}(0, \infty)$ is the consequence of Theorem 11 and Proposition 2 . Indeed, by definition of the spectral function $\varrho(\lambda)$ in this case [17]

$$
N_{n}(\Delta)=\int_{\Delta}\left\{n^{-1} \sum_{1}^{n} u_{k}^{2}(\lambda)\right\} d \varrho_{n}(\lambda)
$$

for any $\Delta=\left(\lambda_{1}, \lambda_{2}\right)$ where $\varrho_{n}(\lambda)$ are the spectral functions of operators $H_{n}$ converging to $\varrho(\lambda)$ as $n \rightarrow \infty$ [17]. The expression in parentheses tends to infinity for almost every $\omega$ as $n \rightarrow \infty$ for almost all $\lambda$. Then, by the boundedness of $N_{n}(\Delta)$ for any $\Delta$ due to the exponential growth of the Cauchy solution $u_{k}(\lambda)$, the set of the growth points of the limit function has a zero Lebesgue measure, hence the spectrum of $H$ has no absolutely continuous component.

\section{Appendix}

Here we sketch the proof of the strict positiveness of $\mathfrak{J}_{ \pm}(\lambda)$ of $(14)$ for the case of $v_{n}$ as a stationary, ergodic and invertible Markov chain. First remind these notions $[5]$. 
Starting with the stationary Markov chain, i.e. a random sequence whose transition probability $P\left(v \mid v^{\prime}\right)=\operatorname{Pr}\left\{v_{n+1} \leqq v \mid v_{n}=v^{\prime}\right\}$ does not depend on $n$, one can construct stationary (one-dimensional homogeneous) sequence if there exists an invariant distribution function $F(v)$ defined by the following equation:

$$
F(v)=\int_{-\infty}^{\infty} P\left(v \mid v^{\prime}\right) d F\left(v^{\prime}\right)
$$

$F(-\infty)=0, F(\infty)=1$. Assume that this integral equation has no nondecreasing solutions but $F(v)$ and that the resulting random sequence is metrically transitive ${ }^{13}$. These two assumptions, generally speaking, are not independent. For instance, if $P\left(v \mid v^{\prime}\right)$ satisfies the Deblin condition, then they are equivalent [5].

A stationary sequence is called invertible if all its finite dimensional distributions

$$
\operatorname{Pr}\left\{v_{k_{1}} \leqq v_{1}, \ldots, v_{k_{l}} \leqq v_{l}\right\}, \quad l=1,2, \ldots
$$

are invariant with respect to transformation $\left(k_{1}, \ldots, k_{l}\right) \rightarrow\left(-k_{1}, \ldots, k_{l}\right)$. For the Markov chain it gives relation

$$
\int_{-\infty}^{v_{1}} P\left(v_{2} \mid v\right) d F(v)=\int_{-\infty}^{v_{2}} P\left(v_{1} \mid v\right) d F(v)
$$

Thus, the operator $\hat{P}$ determined in the space of functions of $v$ which are square integrable with the weight $F(v)$ by the formula

$$
(\hat{P} f)(v)=\int_{-\infty}^{\infty} P\left(v \mid v^{\prime}\right) f\left(v^{\prime}\right) d v^{\prime}
$$

is selfadjoint, $\lambda=1$ being its maximum and simple eigenvalue and the corresponding eigenfunction being a constant (we take it equal to 1).

Since quantity $\mathfrak{I}_{\alpha}(\lambda, \omega, \varphi)$ of Eq. (16), for every $\alpha$ can take on only two values $\pm \mathfrak{I}_{\alpha}(\lambda)$, then $\mathfrak{I}_{\alpha}(\lambda) \geqq E\left\{\mathfrak{I}_{\alpha}(\lambda, \omega, \varphi)\right\}$ and it is sufficient to prove positiveness of $E\left\{\mathfrak{J}_{\alpha}\right\}$. In virtue of stationarity and invertibility of $v_{k}$.

$$
E\left\{\mathfrak{I}_{\alpha}(\lambda, \omega, \varphi)\right\}=E\left\{\mathfrak{I}_{-\alpha}\left(\lambda, \omega, \frac{\pi}{2}-\varphi\right)\right\}
$$

and hence it is sufficient to prove inequality

$$
E\left\{\mathfrak{J}_{\alpha}(\lambda, \omega, \varphi)\right\} \equiv I(\lambda)>0 .
$$

Introduce

$$
\varrho_{k}^{2}=u_{k}^{2}+u_{k+1}^{2}, \quad z_{k}=u_{k} / u_{k+1} .
$$

Then

$$
\frac{\varrho_{k+1}^{2}}{\varrho_{k}^{2}}=z_{k}^{2} 1+z_{k+1}^{2} / 1+z_{k}^{2}
$$

13 The simple sufficient condition for this property is the strict positiveness of the transition probability $P(I \mid v)$ for any interval $I$ 
and

$$
I=\lim _{n \rightarrow \infty} n^{-1} E\left\{\ln \varrho_{n}^{2}\right\}=\lim _{n \rightarrow \infty} n^{-1} \sum_{k=1}^{n} E\left\{\ln z_{k}^{2}\right\}
$$

Introduce the variable $\varphi_{k}$ by relation

$$
z_{k}=\cos \left(\varphi_{k}+\beta\right) / \cos \varphi_{k},
$$

where $\beta$ is a fixed angle. Such a variable for another problem was used in [19]. We have

$$
\begin{aligned}
I & =\lim _{n \rightarrow \infty} n^{-1} \sum_{k=1}^{n} E\left\{\ln \cos ^{2}\left(\varphi_{k}+\beta\right)-\ln \cos ^{2} \varphi_{k}\right\} \\
& =-2 \int_{0}^{\beta} d x \int_{-\pi / 2}^{\pi / 2} \operatorname{tg}(\varphi+x) P(d \varphi),
\end{aligned}
$$

where

$$
P(\varphi)=\lim _{n \rightarrow \infty} n^{-1} \sum_{k=1}^{n} P_{k}(\varphi)
$$

and $P_{k}(\varphi)$ is the distribution function of a random variable $\varphi_{k}$. We obtain from Eq. (13) the recursion relation for $\varphi_{k}$ :

$$
\tan \varphi_{k+1}=\tan \left(\varphi_{k}+\beta\right)+\frac{v_{k}+2 \cos \beta}{\sin \beta}
$$

from which the equation for the limiting joint distribution $P(\varphi, c)$ of the random variables $\varphi_{k}$ and $c_{k}=\frac{v_{k}+2 \cos \beta}{\sin \beta}$ follows :

$$
\int \Phi(\tan \varphi, c) P(d \varphi, d c)=\int \Phi\left(c^{\prime}+\tan (\varphi+\beta), c\right) P\left(d c^{\prime} \mid c\right) P(d \varphi, d c),
$$

where $\Phi(\mathrm{x}, \mathrm{c})$ is a certain (trial) function. From the latter equation and Eq. (19) we obtain

$$
g(o, s, c)=\int e^{i s c^{\prime}} g\left(\alpha, s, c^{\prime}\right) P\left(d c^{\prime} \mid c\right),
$$

where

$$
g(x, s, c)=\int_{-\pi / 2}^{\pi / 2} \exp \{i s \tan (\varphi+x)\} P(d \varphi \mid c)
$$

and $P(\varphi \mid c)$ is the conditional distribution of $\varphi_{k}$ as $k \rightarrow \infty$. It is easy to find that

$$
-\frac{\partial^{2} g}{\partial s^{2}}=-g+\frac{1}{i s} \frac{\partial g}{\partial x} \text {. }
$$

Multiplying this identity by $g^{*}$, subtracting the complex conjugated expression and integrating the resulting relation over $s \in(0, \infty)$ we get:

$$
\left.2 \frac{\partial g^{*}}{\partial s}\right|_{s=0} g(x, o, c)=\left.\frac{1}{i} \frac{d}{d x} \int_{0}^{\infty} \frac{d s}{s}|| g(x, s, c)\right|^{2}
$$


or, after taking into account relations:

$$
\begin{aligned}
g(x, 0, c) & =1,\left.\quad \frac{\partial g}{\partial s}\right|_{s=0}=i \int_{-\pi / 2}^{\pi / 2} \tan (\varphi+x) P(d \varphi \mid c) \\
I & =-2 \int_{0}^{\beta} d x \int_{-\pi / 2}^{\pi / 2} \tan (\varphi+x) P(d \varphi \mid c) F(d c) \\
& =\int_{0}^{\infty} \frac{d s}{s} F(d c)\left\{|g(\beta, s, c)|^{2}-|g(0, s, c)|^{2}\right\} .
\end{aligned}
$$

Equation (21) permits to transform (22) in the following way:

$$
I=\int_{0}^{\infty} \frac{d s}{s}\left\{\|\tilde{g}\|^{2}-\|\hat{P} \tilde{g}\|^{2}\right\},
$$

where $\tilde{g}(s, c)=e^{i s c} g(\beta, s, c)$ and the symbol $\|\cdot\|$ denotes the norm in the space $\mathscr{L}_{2}(-\infty, \infty, F(d c)\}$. Since $\|\hat{P}\| \leqq 1$, then $I \geqq 0$. Our assumption about $\hat{P}$ means that the quality $I=0$ holds if and only if $\tilde{g}$ is independent of $c$. From Eq. (21) it follows that $g(0, s, c)$ is also independent of $c$. Since $g(0, s, c)$ determines $P(\varphi \mid c)$ uniquely, $P(\varphi \mid c)$ and, hence $g(\beta, s, c)$ does not depend on $c$. But $g(\beta, s, c)$ and $\tilde{g}(s, c)$ $=e^{i s c} g(\beta, s, c)$ cannot be independent of $c$ simultaneously.

In the particular case, where $v_{k}$ are independent identically distributed random variables, Eq. (23) takes the form:

$$
I=\int_{0}^{\infty} \frac{d s}{s}|g(\beta, s)|^{2}\left(1-|f(s)|^{2}\right), \quad f(s)=\int_{-\infty}^{\infty} e^{i s c} F(d c)
$$

from which it follows that $I>0$ if $v_{k}$ takes on at least two different values. This result was obtained in [19] by applying some results of [20].

Using similar arguments it can be shown that in the continuous case of the Schrödinger equation, with random invertible Markov potential $v(x)$.

$$
I=\int_{0}^{\infty} \frac{d s}{s}\left(q, L_{v} q\right)
$$

where $L_{v}$ is the infinitesimal operator of the Markov process,

$$
q(s, v)=\int_{-\infty}^{\infty} e^{i s z} p(z, v) d z, \quad z=\psi^{\prime} / \psi
$$

(, ) denotes the scalar product in the Hilbert space $\mathscr{L}_{2}(-\infty, \infty, p(v) d v)$ and $p(v)$ is the density of the stationary distribution $v(x)$.

In a similar way we arrive at an analogous relation for the potentials of the form

$$
v(x)=\sum_{j} u\left(x-x_{j}\right),
$$

where $u(x)=0,|x| \geqq d, l_{j}=x_{j+1}-x_{j} \geqq 2 d$ are independent identically distributed random variables with the density $f(l)(f(l)=0, l \leqq 2 d)$ and for potential $v(x)$ which 
takes on only two values $0, v_{0}>0$ on the random intervals, which are independent random variables with densities $f_{0}(l)$ and $f_{1}(l)$. We have respectively:

$$
I=(a+2 d) \frac{1}{2} \int_{0}^{\infty} \frac{d s}{s} \int_{2 d}^{\infty} d \xi f(\xi)\left|q(s, \xi)-\int_{2 d}^{\infty} d \xi^{\prime} f\left(\xi^{\prime}\right) q\left(s, \xi^{\prime}\right)\right|^{2},
$$

where $a=\int_{2 d}^{\infty} l f(l) d l, F(\xi)=\int_{\xi}^{\infty} f(l) d l$

$$
q(s, \xi)=\int_{-\infty}^{\infty} e^{i s z} p(z, \xi) d z \cdot F(\xi)
$$

$p(z, \xi)$ is the joint probability density of random variables $z(x)=\psi^{\prime}(x) / \psi(x)$ and $\xi(x)$, the latter being the separation of $x$ and $x_{j}$ which is the closest to $x$ on the left;

$$
I=\left(a_{0}+a_{1}\right) \sum_{r=0,1} \int_{0}^{\infty} \frac{d s}{s} \int_{0}^{\infty} d \xi f_{r}(\xi)\left|q_{r}(s, \xi)-\int_{0}^{\infty} d \xi^{\prime} f_{r}\left(\xi^{\prime}\right) q\left(s, \xi^{\prime}\right)\right|^{2}
$$

where

$$
q_{r}(s, \xi)=F_{r}(\xi) \int_{-\infty}^{\infty} p_{r}(z, \xi) e^{i s z} d z .
$$

$p_{\boldsymbol{r}}(z, \xi)$ is the density of random variables $\xi_{\boldsymbol{r}}(x), z(x)=\psi^{\prime}(x) / \psi(x), \xi_{r}(x)$ is the separation of $x$ and the left-neighbouring jump of $v(x)$,

$$
a_{r}=\int_{0}^{\infty} l f_{r}(l) d l, \quad F_{r}(\xi)=\int_{\xi}^{\infty} f_{r}(l) d l
$$

Acknowledgement. I am indebted to Professors V. A. Marčenko and Ya. G. Sinai for the stimulating discussions, Dr. I. Ya. Goldsheidt for the improvement of the first proof of Theorem 9 and Dr. V. A. Tkačenko for many helpful remarks.

\section{References}

1. Lifshitz, I.M.: Adv. Phys. 13, 485-520 (1964)

2. Mott, N.F., Davis, E.A.: Electronic processes in monocrystalline materials. Oxford: Clarendon Press 1971

3. Thouless, D.J.: Phys. Rep. 13, 93-142 (1974)

4. Pastur, L.A.: Usp. Mat. Nauk. 28, 3-64 (1973)

5. Doob, J.L.: Stochastic processes. New York: Wiley 1953

6. Pastur, L.A. : On the spectrum of the random Jakobi matrices and the Schrödinger equation on the whole axis with random potential. Preprint, Kharkov (1974)

7. Goldsheid, I.Ya., Molchanov, S.A., Pastur, L.A.: Funksionalny Analiz i yego Prilozheniya 11, 1-10 (1977)

8. Kato, T.: Perturbation theory for linear operators. Berlin, Heidelberg, New York: Springer 1966

9. Grenander, U., Szegö, G. : Töplitz forms and their application. Los Angeles: University California Press 1958

10. Besicovitch, A.S.: Almost preriodic functions. Cambridge: Cambridge University Press 1932

11. Anderson, P.W.: Phys. Rev. 109, 1492-1505 (1958)

12. Fujita, S. : Introduction to non-equilibrium statistical mechanics. London: W. Saunders C. 1966

13. Loomis, L.: Trans. Am. Math. Soc. 53, 239-248 (1943) 
14. Reed, M., Simon, B. : Methods of modern mathematical physics. IV. Analysis of operators. New York: Academic Press 1977

15. Casher, A., Lebowitz, J.: J. Math. Phys. 12, 1701-1711 (1970)

16. Ishii, K. : Progress Theoret. Phys. Suppl. 53, 77-138 (1973)

17. Berezanski, Yu.M.: Razlozheniya po sobstvennym funktsiyam samosopryazhennykh operatorov. Kiev: Naukova Dumka 1965

18. Oseledets, V.I.: Tr. Mosk. Mat. Ova. 17, 179-210 (1968)

19. Matsuda, H., Ishii, K.: Progr. Theor. Phys. Suppl. 45, 56 89 (1970)

20. Furstenberg, H.: Trans. Am. Soc. 108, 3-28 (1963)

21. Gredeskul, S.A., Pastur, L.A.: Sov. J. Low Temp. Phys. 1, 135-152 (1975)

22. Lifshitz, I.M., Gredeskul, S.A., Pastur, L.A.: Sov. J. Low Temp. Phys. 2, 533-549 (1976)

23. Shubin, M.A.: Usp. Mat. Nauk. 32, (2), 95-135 (1979)

24. Marchenko, V.A., Pastur, L.A.: Mat. Sb. 72, 507-536 (1967) [Mat. USSR, Sbornik, 1, 457-483 (1967)]

25. Dynaburg, E.I., Sinai, Ya.G. : Funkts. Analiz Priloz. 9, 8-21 (1975)

26. Kunz, H., Souillard, B.: Random Schrödinger operators and the theory of disordered systems: some rigorous results. Preprint, Palaiseau (1979)

27. Belokolos, E.D.: Theor. Math. Phys. 25, 344-357 (1975) ; 26, 35-41 (1976)

Communicated by Ya. G. Sinai

Received October 10, 1979 